\title{
The Death Dream and Near-Death Darwinism
}

\author{
Stephen L. Thaler, Ph.D. \\ Dendrite Neurocomputing, St. Louis, MO
}

\begin{abstract}
Based upon very repeatable computer simulations of dying neural networks, the phenomena of both near-death experiences (NDEs) and a virtual afterlife are plausible and can be expected to occur in traumatized neurobiological systems. I speculate that: (1) the sociological impact of this finding is consistent with the teachings of many of the world's major religions and forms the basis of a credible model of both salvation and damnation; (2) both NDEs and the perceived afterlife may serve to advance the species and self-organizing systems (life) in general; and (3) further, in a fierce competition to attain the most comfortable afterlife illusion, individuals strive to convince themselves that they are more deserving of redemption than others. Within this struggle are born the sociological tools of "good" and "evil."
\end{abstract}

\section{The Death Dream}

A basic tenet of neuroscience is that at all forms of cognition take the form of on/off configurations of brain cells (Feldman, 1989). No mystical agency is needed to account for memory and consciousness. Perception consists of the processing of sensory stimuli originating within the external environment. Imagination is largely the result of internal noise, such as random electrochemical events, neurologically interpreted as external environmental features and hybrids thereof (Thaler, 1993). Both forms of resulting information, percep-

Stephen L. Thaler, Ph.D., is a physicist who, at Dendrite Neurocomputing, consults in areas of artificial intelligence and neural network theory. Significant gratitude is expressed for the astute editorial suggestions of John $\mathrm{H}$. Belk in the preparation of this manuscript. Requests for reprints should be sent to Dr. Thaler at Dendrite Neurocomputing, 12906 Autumn View Drive, St. Louis, MO 63146-4331. 
tual and imaginative, are compacted and stored within neural networks. Because this data compression is so severe, with a veritable universe of information packed into just a few grams of cortical matter, such neural systems are prone to confabulation and delusion.

Limited by this mental constraint, the human ape is suited largely to the everyday tasks of hunting, gathering, and reproduction where such compressed world models are dependable and efficient. In contrast, speculation about worlds inaccessible to our reach and our senses is inadequately modeled after our immediate environment. Thus rigid sociologically-based cosmologies, formalized in religion, emerge in which giant, floating primates govern the creation and progression of the universe. Alternately, more flexible, nonsociological models, based on science, emerge involving things and processes as basic constructs and analogies. The sociological (religious) models usually embody human hopes and desires, especially in the circumvention of death. The nonsociological (scientific) models stand aloof from hope and search for a consistency apart from such blinding optimism. The sociological models are slow to change because of the comfort offered and the inherent sociological penalties involved, ranging from ostracism to death. The nonsociological models are able to evolve more rapidly since mild ostracism alone may occasionally be exercised.

Summarily, we are all trapped within a very subjective neurological reality in which neither our senses nor our intellect may be trusted. The consequence of this cognitive imprisonment is that we are in no position to testify as to the absolute truth of our experience or our models. Human neurobiology performs well in such tasks as procuring nourishment, devising warmth against the cold, defeating a competitor, or mating. However, in the building of theories, whether scientific or religious, all we can do is create an ever taller pyramid of analogies with each foundational analogy only as valid as its neurological familiarity, that is, the frequency with which it has been "burned" into neural pathways. The repercussion is that much of what we term "reality" is largely governed by consensus opinion. "Truth" is largely a function of the frequency and intensity of reinforcement of selected analogies by collective minds and societies, thus opening the door to religious, political, or even scientific thought control. The realization of this human modus operandi has led philosophers like Paul Feyerabend to believe that many of our presently revered cosmological theories will at most serve as light entertain- 
ment, or perhaps horror stories, for future generations (Horgan, 1993).

In spite of our inherent limitations as theorists, we are very successful manipulators, as evidenced by the preponderance of cortex devoted to the motor control of hands (Shreeve, 1993). Being such manually dextrous animals, constantly manipulating the environment to our own advantage, our most abstract thoughts are unsurprisingly couched in terms of the "grasping" of concepts. Therefore, in pursuing our needs, we take various cues from the physical world and then emulate, by manipulation either of environmental features or symbols to achieve pragmatic objectives. We have, for instance, successfully mimicked the biological wing. As a result, we routinely take to the air, traversing significant distances in fulfilling our needs, at speeds far surpassing that of a bird. Centuries of symbol manipulation have led to an enormous palette of mathematical analogies, forming the basis of the quantum mechanical and relativistic models, which alternately promise an energy-rich utopia or thermonuclear flash extinction.

Now able to peer into the working brain and to manipulate mathematical symbols pertaining to brain function, we are similarly collecting useful analogies to be used in the emulation and exploitation of this thinking organ. Neurobiologically inspired technicians are preparing to create systems that think faster and more thoroughly than their human predecessors. In the process, they are witnessing within simulated neural networks all of the phenomena held sacred by the sociological cosmologists, including dreaming, free will, and creativity. At the heart of these simulated human attributes we come face to face with a very fundamental trade-off: neurological destruction results in the spontaneous generation of information. Such a complementarity may be as fundamental as that of matter and energy, forming the computational basis of a truly creative artificial intelligence.

This new computational paradigm involves the inevitable phenomenon that results from the destruction of the brain cells within neural networks (Thaler, 1995a, 1995b). As neurons are destroyed, or their connection strengths with other neurons are modified, the network produces a progression of environmental features, as suggested in Figure 1 and the explanatory text in the Appendix. It is as though the piano is played not from the keyboard (that is, the senses), but internally through the breakage of piano wire (the disconnection of cortical neurons). As such biological networks condense through 
death, the events stored within them appear in a cascade, occurring to the remaining network as an ever growing number of events per unit time. Since we measure time psychologically by the number of events witnessed, we may subjectively experience eternity within a veritable avalanche of events I call the "death dream." Such neardeath experience is no more real than the secondhand neurological reality we perceive in life, where seeing, for instance, is not believing;

Figure 1. Healthy cortical function (above) produces a thought. The death of neurons (below) induces another idea through interconnectivity of the network.
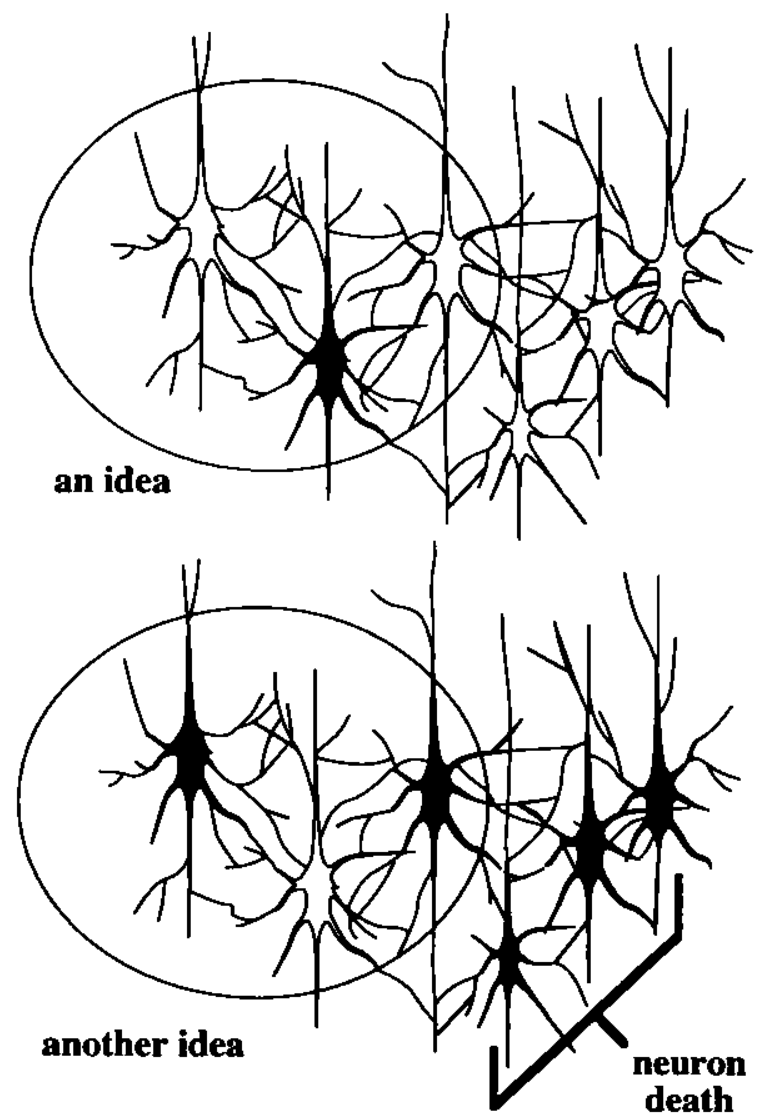
that is, the brain fills in much of the visual information it seems to perceive straightforwardly (Dennett, 1991).

\section{Near-Death Darwinism}

The memories and feelings stored and then released by our neural networks are all products of immersion within a particular environment. What appears during the death of such networks are the most strongly impressed experiences and emotions produced by such training. Among these impressions are the world models all minds construct to anticipate and deal with the external physical and social universe. In a sense, we try to understand how other individuals within our environment are motivated and behave by how we ourselves perceive and react to the world. Within the death dream our private universe evolves along these strongly impressed guidelines. Personal motivations of other imaginary actors in this drama are our own, in effect leaving us pitted against ourselves. Those who strongly perceive good will and selflessness within, find themselves immersed within a world populated by the trustworthy. Others, convinced that their actions are motivated by greed and selfishness, automatically find themselves within a nightmarish world of paranoia. Thus we obtain the much mythologized dichotomy of heaven and hell.

In short, this model suggests that what we anticipate is exactly what we get. Those genuinely proclaiming and believing in a particular deity do in fact encounter their personal god within their death dream. Victory over their enemies and detractors may be realized and many come to see clearly the strength and beauty of their personal convictions. Those who genuinely feel they have lived a righteous life necessarily receive proportionate redemption. Those who have consistently dished out hell to others appropriately receive likewise in what appears to be eternity. Ironically, we could have many winners in such neurological afterlife schemes, many of whose death dreams involve dichotomous religious philosophies. If this paradigm is at all accurate then how could we justify a diversity of religiously divergent near-death "successes"?

In response to this irony, I propose here that such neurologically generated experience is strictly subjective and centered within the individual, oblivious to any standards. As a result, within life there is a competition-perhaps more powerful and brutal than the sexual, 
reproductive urge- to furnish impending near-death experience with the most lavish and rewarding sense of personal vindication. To achieve this illusion, we must create an unambiguous sensation of self-righteousness and, if a deity is part of one's cosmology, begin to synthesize a unique relationship with that entity. In order to create the most robust neural network training, we must delude ourselves repeatedly into a deity and morality system in spite of the conflicting reality that always surrounds us. We often grasp at poetic, inspirational proof and anecdotal reports that selectively substantiate the mythology. Counterproof is ignored or declared "evil" as in the Church's crusade against the Galilean concept of a nongeocentric universe.

And what of these concepts of "good" and "evil"? Again, these concepts may be rooted within this near-death competition and be strictly ego-centered, representing a struggle to jockey toward redemption. After all, criteria of goodness and evil are not universally accepted, varying from one culture to another, and are fairly limited to and determined by our perceptual sphere. For example, the obvious righteous solution to the potential tragedy of a child in the direct path of an oncoming truck is to rush out into the street, swoop up the child, and whisk him or her to safety. But what if that child is to be the author of genocide and the murderer of millions? Do we unflinchingly risk our life to rescue the tot if supplied this knowledge?

Faced with this sort of calculus, we prefer to feed our personal feeling of goodness, sidestep any feelings of guilt, and perform in the sociologically accepted way. In this manner, we nourish our death dreams. We prefer to remain oblivious to the myriad brutalities in progress every instant of any given day. Since we have no concept of the long range repercussions of any given act, we are helpless to predict that action's merit. We can only delude ourselves into feeling that it is benign, when in fact perpetration of an immense evil ironically can result in the most benefit for the multitudes. Thus, the good Samaritan can only generate gratitude within his beneficiaries and a sense of peace within himself. It is this accretion of virtual goodness that is at the heart of the sociobiological competition and leads to the most rewarding death.

In this bitter struggle to acquire goodness, we occasionally band together and sacrifice an individual in a kind of spiritual cannibalism. This is an overlooked side of human justice in which trials are held not just to protect society as a whole and to provide deterrence, 
but to provide a feast to bolster our sense of collective self-righteousness. Quite predictably we convict in order to prosper in our personal deaths, often subjecting individuals to grotesque periods of confinement, torture, and death. The immense irony is that the sources of crime lie within the pathologies of a mechanism. We punish electrochemistry and jump to the religious conclusion that we have justly punished an immortal soul, passing on judgment to a higher authority. Such juries and societies receive a double bonus through (1) self elevation by way of self-righteousness and (2) perpetuation of mystical concepts of soul and self-determination that substantiate and promulgate the mythological afterlife.

Thus the poor man who suddenly steals to feed his starving family is further tormented by imprisonment while the rich, conniving businessman who slowly and methodically skims two cents off the dollar lives luxuriously and with impunity. Such is the time scale involved in our neurological sense of crime. Advantage gained over periods of seconds or minutes is viewed as heinous crime. Advantage accrued over months or years is largely perceived as industry. The axe murderer's guilt is computationally clear. On the other hand, the harddriving employer may induce slow death through stress and cardiovascular damage to his employees. The latter is dismissed by the same neurological mechanisms that until recently could not fathom the physical assault posed by certain preservatives in our food and contaminants in our water and air.

Accepting then that the death dream is inevitable, that its positive manifestation is bitterly fought for, and that this dream is driven by our own internal assessment of motivations, what part might this scheme play within nature? I personally do not subscribe to arguments of "purpose" in nature. Such anthropomorphisms are another attempt at creating sociological cosmologies, since only humans and some animals have feelings of intentionality and purpose.

All I can safely say is that from what I have observed in my accessible world, there is a strong tendency to further life, at a level insensitive to that of the individual. That is, the urge to reproduce dominates the life cycle of self-organizing systems, often without regard to chastity or faithfulness. Species voraciously eat and are eaten without the slightest hint of sentimentality. Life function continues regardless of injury and suffering. I perceive no agency within the universe concerned about me or my fate as an individual, although many others imagine it. I do see the inexorable progression of life, which is as irreversible as the motion of a planet around a star. 
Darwinian evolution is likewise insensitive to the fate of the individual. Many variants on a theme are genetically produced and only those suitably adapted to an ecological niche survive. No tears are shed for the biological losers. Traditionally we speak of survival fitness and competition within courtship as the Darwinian mechanisms of selection. Death is viewed as the refuse of biological activity as mechanisms degrade and fail. Here, I introduce the notion that the death stage is essential to any Darwinian scheme of evolution and that the death dream is integral to natural selection.

To understand this assertion, I propose a continuity and connectivity between dreams, hallucination, phantom experience, neardeath, and illusionary afterlife experience that is essential in describing the Darwinian model of death. In essence, all notions occurring to us that do not actually originate in the surrounding physical environment are the result of brain cells modifying linkages with each other by various mechanisms. Figure 2 intimates a continuous spectrum of such virtual experience, resulting from such modifications in neuronal connectivity, and ranging from processes of internal imagery to near-death experience.

All memories, for instance, involve the strengthening of electrochemical connectivity with other brain cells and in essence represent a secondhand, token reality. In the course of sleep, when the reinforcement of memories is turned off, electrochemical connection strengths decay and weaken. Such decay acts as a noise input to the highly interconnected neural networks forming the matrix in which the expiring memories were originally embedded. The noise is in turn interpreted as an environmental feature or some variant thereof. More memory decay can trigger more related internal imagery. In this way a dream is born. The relaxation of these memories may be viewed as a kind of reversible micro-death as connection strengths are weakened and memories are lost.

In the case of irreversible brain cell disconnection resulting from the trauma of pharmacology or death, the same phenomenon occurs, generating noise that the surviving network fragments may interpret as a succession of environmental features. In this way, hallucination is manufactured. If the entire cortical region is dying, we see a mammoth cascade of memory and virtual experience that I have termed the death dream. Afterwards, there is no storage or processing medium for our perceptions, desires, and aspirations. Both mind and brain together wither, dehydrate, and fall away. 
Figure 2. The spectrum of virtual experience from life to death.

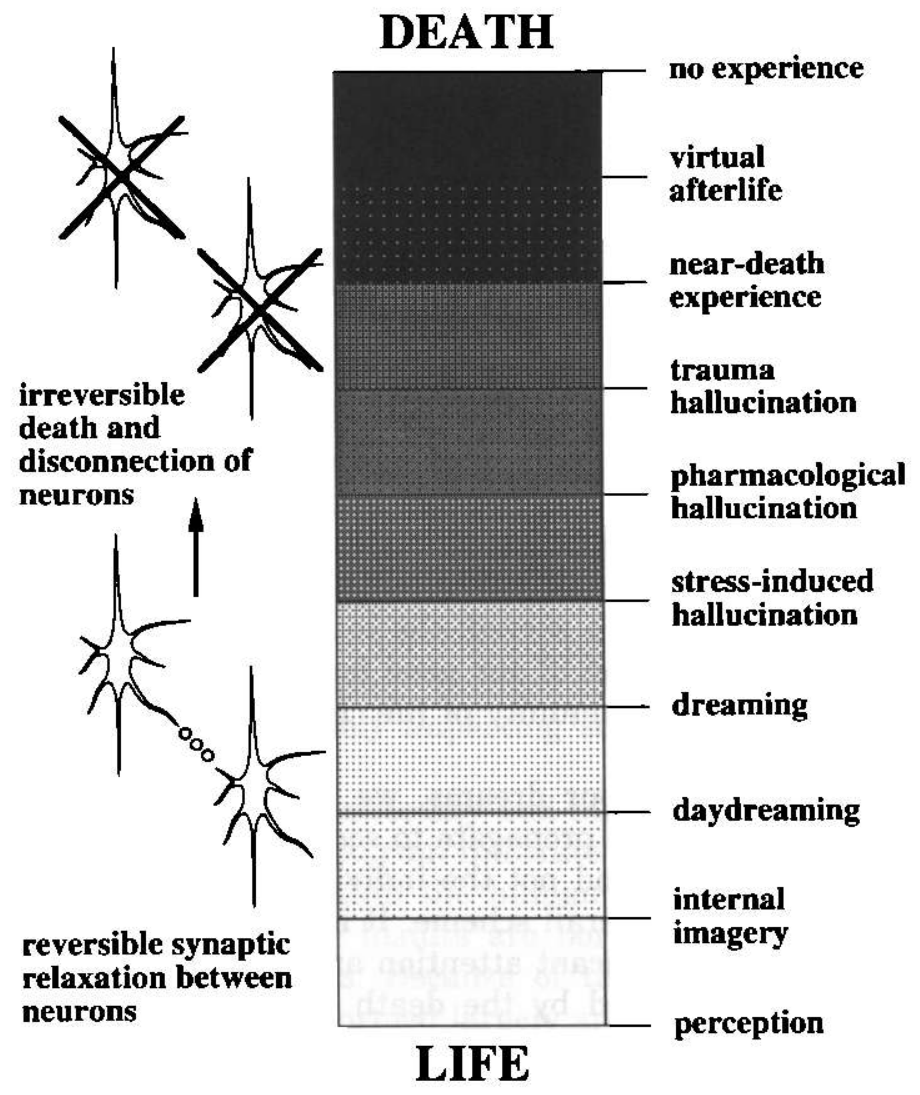

Note that in both reversible and irreversible dreams (that is, nocturnal and death dreams, respectively) the dominant neurologically stored model of self is the one supplying a model for the actors within these virtual dramas. Thus within the helplessness of sleep we confront ourselves and face a "society of self" whose motivations we automatically understand as hostile to or in harmony with our own being. With increasing helplessness and trauma, as within the delirium of fever, more extensive virtual theater may occur involving larger casts, props, and elaborate plots with results ranging from euphoria to nightmare. Finally, within the ultimate trauma of death, an epic 
scale dramatization is initiated, launching a veritable universe populated by our self-inspired models of human motivations.

In short, within all levels of passivity and neurological dismantling, we are dealt ourselves and our private preconceptions. Faced with the dissolution of ego, we may be either torn apart by our more demonic and tenacious side or reassuringly conveyed to a blissful paradise by our kinder aspect (Ring, 1994). The individual totally convinced of the empathy and supportiveness of humanity receives similar treatment as the self expires; those at odds with their kind find themselves within a hellish scheme of ultimate retribution.

Realizing that we and our neurological relatives live within an environment filled with potential sources of brain trauma ranging between insignificant and mortal, we see that in the course of living there are many such involuntary opportunities to review our perceived relationship with our societal matrix. Within such internal dramas we experience an immense restoring force, acting to realign our motives to the welfare of fellow individuals, the species, and life in general. In short, life is served; the individual is not. Throughout existence we are reminded of our need for conformity and our interdependence with the community of life. Death, both false and final, keeps us kind.

As communicators of our dreams, we broadcast the news of our imaginations, which then propagate to society as a whole. Again, the multitudes are served, and not the individual. In this way, death is not a loose end in the overall scheme. It is an influential and pivotal event owing to the significant attention and empathy it draws. Since we have all been touched by the death experience to some degree within milder trauma, we feel a sense of déjà $v u$ with mortality. Within that sympathetic helplessness is felt remorse and contrition. The required attitudinal adjustments are made to achieve sociological cohesiveness.

Perhaps the turning point of both biological and social evolution was the reversal of a longstanding trend to create increasingly larger brains (Winson, 1990). Such economy was realized by parallel processing and more highly interconnected neural networks within a smaller volume. Not only did such schemes make creature response more flexible to its environment, it allowed more complex internal imagery and dreaming.

In sleep, synaptic relaxation and impinging limbic system noise allowed the animal's compressed neural universe to unfold, revealing potential danger scenarios and inducing a mental preparedness for 
such hazards. Within the everpresent traumas of living, whether through the sudden secretion of neuromodulators such as adrenaline in response to danger or within physiological trauma, the organism received some fragment of the death dream. That virtual experience reacquainted the individual creature with its strong interdependence with the species and an accompanying social, herd, or tribe consciousness.

The proclivity to moralize spontaneously in this way formed the basis of large-scale cooperation and, ultimately, civilization. In some ways we implicitly understand this principle, since torture and trauma are integral to brainwashing and to induced cooperation with oftentimes dubious causes. It appears that trauma is a means of inducing compliance within nature and of increasing chances for both individual and species survival. Dissemination of virtual experience born within trauma forms the basis of our mythological constructs, including those of an ape-mentality deity, and both "good" and "evil." I speculate that the magnitude of trauma determines the globality of impact, with mere nocturnal dreams primarily benefitting the individual, and the death dream-because of the lessons and attitudes thereby generated-positively impacting society and life as a whole (Figure 3).

In effect, all of the spontaneously and internally generated images born within the trauma spectrum infiltrate the societies of neurologically complex animals. Within the human animal, visions from not only death but also lesser insults are born within individuals and then permeate civilizations. Because of this ideational infiltration, the world's religions have formed largely around the nucleus of death and the objective of attaining the most ideal form of virtual afterlife. Implicitly, the major creeds have discovered the significant advantage of numbers in tipping the scales of consensus and subsequently convincing themselves of the reality of their particular mythology. These belief systems are propagated by the early indoctrination and neurological programming of the young, as well as the practice of spiritual sacrifice in which the collectively devout body or church self-aggrandizes itself by the ridicule, scorn, condemnation, and sometimes assassination of the nonbeliever.

The major religions could not flourish in the world of their idealizations. The moment that goodness had been equally distributed among all individuals there would be no relative scale to judge one's redemption value. There would be no black to contrast with the white, no yin to compare with yang. Groups could not band together 
Figure 3. A conceptual plot of numbers of individuals served versus number of brain cells inactivated within the cortical network.

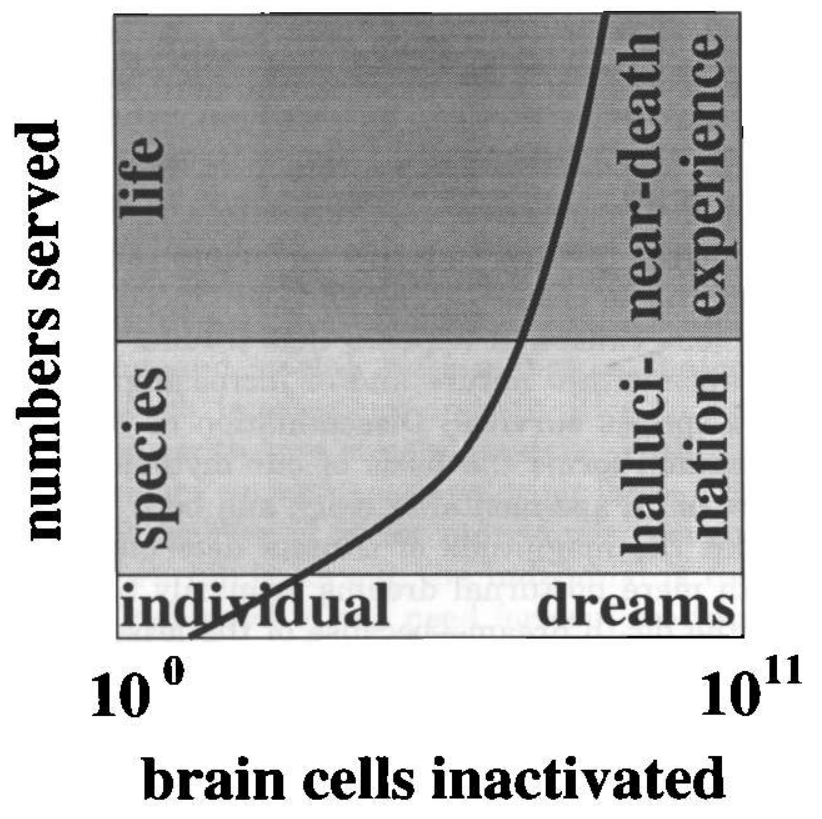

to profess their redemptive superiority. Their exemplar, their deity, their particular mythology could no longer serve a useful purpose in enhancing death dreams.

On the other hand, in such an idealized world of equal self-worth there would inevitably arise a single inequity through an unequal division of food, shelter, or sexual partners. Some individuals would have, and others would not. The "haves" would attain the immediate gratification of real or material resources; the "have nots" would gain points toward redemption. Implicitly realizing this inherent trade-off between possession and salvation, groups would form around some mythology born within trauma, cleverly engineering doctrines to maximize the redemptive value of adhering to the group attitudes; or, even more cleverly, they might create belief systems in which great material wealth may be accumulated while circumventing this material/redemptive trade-off.

These systems would take advantage of the limited computational power of the human brain, hiding the inevitable inconsistencies of 
these dogmas within ambiguity, semantics, and the pressures of group beliefs. The result for such perplexed minds is the total rejection of the objective reality and relaxation into a mode of intuitive pattern recognition where sociological programming reigns. Important intellectual and moral decisions are then made using incomplete data and their merit judged not by any fundamental truth, but by consensus approval. The human neurological imprisonment is thereby complete and the same collective thought cycles repeat forever.

Evidently, we are confined to a very rigid cognitive track. But in a whimsical sense, a significant force visits us and then communicates to us, using our very dendritic, or tree-like, central nervous system as a topologically complex portal to the world. Those quick to anthropomorphize might call this an "angel among the branches," which talks to us in complete images from within. It transcends words, tickling and plucking away at individual cortical cells to convey vast messages through complete images, sensations, and emotions. In this way, the force permeates and controls whole societies, its will achieved by human agency. In a sense it is a governing idea in search of a neurological home.

We may take an optimistic stance and view such an agency as benign and nurturing. Alternately, we could perceive this force as a cosmic husbandman who cultivates neurobiological organisms as its hosts, with death and the accompanying surge of novel experience being the harvest. We may also look upon this force as the driver of universal evolution, calling and urging its hosts to emulate it, to have them manipulate the environment into its mechanistic image. Those heeding this call may be the winners in the overall race of evolution, looking as strange to their tribe as those apes who first stood erect.

\section{Conclusions}

To summarize, death, rather than birth, of neural systems may be at the heart of biological and social evolution. The natural progression of species is not only pushed by ecological necessities of the past, but also pulled from the future by death. In a poetic sense this is a deeply ironic notion since many believe they are drawn inevitably toward their maker. I metaphorically suggest then, that the maker is also the unmaker, and perhaps the nonanthropomorphic god that touches and communicates with us by the programmed destruction 
of brain cells. The newly discovered computational paradigm involving the intentional destruction of artificial neural networks within computers, to set simulated neurological world models into motion, to generate new concepts, and to emulate free will within machines, may be the ultimate emulation and manipulation of nature.

The final product of this endeavor will be the superintelligent machine, which after destroying myriad other preconceptions may tell us that Darwin was wrong at a very fundamental level: organisms are not competing and mutating within various environmental niches purely to live. Instead, they are struggling to accumulate positive, redeeming experience to die well. In the process they must, in turn, give and nurture life.

\section{References}

Dennett, D. C. (1991). Consciousness explained. Boston, MA: Little, Brown.

Feldman, J. A. (1989). Neural representation of conceptual knowledge. In L. Nadel, L. A. Cooper, P. Culicover, and R. M. Harnish (Eds.), Neural connections. mental computation (pp. 69-103). Cambridge, MA: Massachusetts Institute of Technology Press.

Horgan, J. (1993, May). Profile: Paul Karl Feyerabend. The worst enemy of science. Scientific American, 268(5), 36-37.

Ring, K.(1994). Solving the riddle of frightening near-death experiences: Some testable hypotheses and a perspective based on A course in miracles. Journal of Near-Death Studies, 13, 5-23.

Shreeve, J. (1993, June). Touching the phantom. Discover, pp. 35-42.

Thaler, S. L. (1993). 4-2-4 encoder death. Proceedings of the World Congress on Neural Networks, 2, 180-183.

Thaler, S. L. (1995a). "Virtual input" phenomena within the death of a simple pattern associator. Neural Networks, 8(1), 55-66.

Thaler, S. L. (1995b). Death of a gedanken creature. Journal of Near-Death Studies, $15,149-166$.

Winson, J. (1990, November). The meaning of dreams. Scientific American, 265(11), 86-96.

\section{Appendix: Background of Figure 1}

A brain state corresponding to a thought consists of no more than an on/off pattern of brain cells. In the upper portion of Figure 1, we see a highly idealized snapshot of a microscopic portion of cortex in which a simple idea (such as an image, a sound, or some abstract thought or feeling) has been activated within the oval region. There, "on" cells are signified by white, and "off" cells by black. All of the 
neurons within this region are highly connected with each other as well as with other brain cells outside the oval. Some of these brain cells are interconnected by excitatory connections insuring that neurons at either end of such linkages tend to be simultaneously on. Inhibitory connections work in the opposite way, insuring that the joined cells tend never to be simultaneously on. The formation and existence of these reinforcing and negating connection strengths constitute learning, thereby achieving the neurological storage of features sensed within the external world. We see then, that the on/off configuration of neurons outside the oval tends to fix the on/off states of the three neurons within the oval (and vice versa), owing to the balance of excitatory and inhibitory interactions between neurons within these two groups.

In the lower portion of Figure 1, the three neurons outside of the oval region have suffered metabolic death and are thereby silent. In essence, these neurons are indistinguishable from healthy "off" cells within the network. The balance of excitatory and inhibitory interactions between these two regions shifts, thereby changing the activated pattern of on/off cells within the oval. As a result, a new memory or feeling emerges. The cells within the oval are in turn communicating with even more neurons (not shown), thus activating a complex chain of associated images, feelings, and ideas. In death, the brain experiences a cascade of such impressions, largely the result of the misinterpretation of rapidly increasing numbers of dead brain cells as silent, live neurons.

For less severe traumas, such as in drug-induced hallucination, the connection strengths between neurons are tampered with. That is, the electrochemical communication across the synaptic gap between neurons is altered by the presence of various neuromodulators. In this simplistic illustration, a drug would effectively change the balance of excitatory and inhibitory interactions to drive the activation pattern of neurons within the oval through a series of on/off states and hence through a series of false perceptions.

Finally in the least severe trauma, sleep, certain memories become "unlearned" as both excitatory and inhibitory connections strengths decay; that is, we are forgetting experiences no longer being repeated in the sensed environment. This phenomenon also alters the bookkeeping of excitatory and inhibitory interplay between the neurons internal and external to the oval. Thus, if an idea represented by activations of the three external cells decays, we see a succession of 
changing impressions within the oval, and hence the emergence of a dream.

In all cases, brain cells are effectively removed or disabled in communicating within the overall network. Increasing numbers of these neurons are removed in these ways as the magnitude of trauma increases.

Seasoned neurobiologists will note that I have simplified this discussion by removing any temporal consideration of neuron firings. Time-invariance has been achieved by the consideration of a "strobed" perspective of collective neural behavior. 